Kam-Fook Tse

Nagoya Math. J.

Vol. 48 (1972), 19-36

\title{
NEO-NORMAL FUNCTIONS IN ARBITRARY REGIONS
}

\author{
KAM-FOOK TSE
}

\section{Introduction}

It is well known that many properties possessed by functions holomorphic and bounded in a region are also possessed by functions meromorphic and omitting three values. Noshiro [14] in 1938 and Lehto and Virtanen [12] in 1957 independently defined the notion of "normal functions"; they and many others subsequently discovered that most properties concerning boundary behavior and value distribution acquired by meromorphic functions omitting three values in the unit disk (or more general, in a simply-connected region) are also valid properties of "normal functions" defined there. In their research on the problems of value distribution of normal functions, Lange [9], Gavrilov [5] and Gauthier [4] have discovered that functions normal in the disk are exactly those which omit three values "locally," i.e., they do not possess any " $p$-sequence" (see above references). However, the definition of a function being normal in a region depends on the simply-connectedness of the region or its universal covering surface. It is thus difficult to judge if a function defined in an arbitrary region is normal.

In this paper, we shall try to generalize the notion of "normal functions" by using some easy-to-recognize terms and metric-like functions. It is obvious that many theorems, especially those concerning boundary behavior for normal functions defined in the disk, do not hold true or do not even make sense for "normal functions" defined in an arbitrary region. However, a number of theorems concerning value distribution do hold for "normal functions" defined in an arbitrary region.

Throughout this paper, we shall use $G$ to denote a region in the complex plane having at least one finite boundary point except otherwise specified. If a region has no finite boundary point, then it is simplyconnected of parabolic type. Lehto and Virtanen in [12, Sect. 11, p. 55]

Received September 29, 1971. 
showed that only constant functions are normal there and we will not look into this here. (Also see Yosida [19].)

To characterize the criterion of value distribution of "neo-normal" functions in $G$, we shall, in section 2 , introduce a neo-metric in $G$. In fact, this neo-metric will help us to have a better understanding of nonEuclidean metric "in small" in simply-connected regions of hyperbolic type.

In section 3, we shall define the notion of "neo-normal" functions in arbitrary regions. Some necessary and sufficient conditions will be explored, and some remarks will be made on the equivalence between our definition and the previously defined ones for functions normal in a simply-connected region.

In section 4, we shall look into the problem concerning the growth of the derivatives of neo-normal functions. For the case in which $f(z)$ is a holomorphic function bounded or omitting three values in the disk, the study of the growth the quantity " $\left(1-|z|^{2}\right)^{k}\left|f^{(k)}(z)\right|^{\prime}$ near the boundary have been extensively investigated by Seidel and Walsh [16]. The same problem has also been investigated by Rogosinski [15] for bounded functions defined in an angle, and by Eggleton [2] and Xou (Tsou) [18] for bounded functions in arbitrary regions. We shall generalize their results to "neo-normal functions."

Finally, in section 5, we shall make a remark concerning the connections of our results and the studies of Lehto [11], Lehto and Virtanen [13] and Gavrilov ([6], [7], [8]) on meromorphic function in a deleted neighborhood of one of its isolated singularities.

In the sequel, let $G$ be defined as before and $\partial G$ be its boundary. If $z \in G, 0<\theta<1$, then $d(z)=\min \left\{\left|z-z^{\prime}\right|: z^{\prime} \in \partial G\right\}$ and $G(z, \theta)=\left\{z^{\prime} \in G\right.$ : $\left.\left|z-z^{\prime}\right|<\theta d(z)\right\}$. We shall use $C, D, \mathscr{C}, \mathscr{R}, \Omega, d$ and $\chi$ to denote the unit circumference, the unit disk, the complex number plane, the real number axis, the Riemann sphere with radius 1 , the Euclidean metric on $\mathscr{C}$ or $\mathscr{R}$, and the chordal metric on $\Omega$, respectively. If $G$ is a simplyconnected region of hyperbolic type, then $\rho$ will denote the non-Euclidean metric in $G$ and for $0<r \leq \infty, D_{\rho}(z, r)=\left\{z^{\prime} \in G, \rho\left(z, z^{\prime}\right)<r\right\}$. In particular, if $G=D, z_{1}, z_{2} \in D$, then

$$
\rho\left(z_{1}, z_{2}\right)=\frac{1}{2} \log \frac{\left|1-\bar{z}_{1} z_{2}\right|+\left|z_{1}-z_{2}\right|}{\left|1-\bar{z}_{1} z_{2}\right|-\left|z_{1}-z_{2}\right|} .
$$


Moreover, a saquence of points $\left\{z_{n}\right\}$ in $G$ is said to be a boundary sequence of $G$ if $\left\{z_{n}\right\}$ has no limit points in $G$. On the other hand, $\Gamma$ is said to be a boundary path if $\Gamma=\{z=z(t): 0 \leq t<1\}$, where $z(t)$ is a continuous function from $[0,1)$ to $G$, with $\lim \sup \{z(t): t>1-1 / n\}$ $\subseteq \partial G$. Finally, if $f(z)$ is a meromorphic function in $G$, then $\rho(f(z))=$ $\left|f^{\prime}(z)\right| / 1+|f(z)|^{2}$ and we say that $f(z)$ has "Picard behavior" in a set $A$ of $G$ if $f(z)$ assumes every value, except perhaps two, in $\Omega$, infinitely often in $A$.

\section{A Neo-metric in $\boldsymbol{G}$}

Definition 2.1. We define $s: G \times G \rightarrow[0,1]$ by the following:

(i) If there exists $\theta_{1}, 0<\theta_{1}<1$, such that $z_{1} \in G\left(z_{2}, \theta_{1}\right)$ and $z_{2} \in G\left(z_{1}, \theta_{1}\right)$, then

(2.1) $s\left(z_{1}, z_{2}\right)=\inf \left\{\theta: 0<\theta<1, \quad z_{1} \in G\left(z_{2}, \theta\right)\right.$ and $\left.z_{2} \in G\left(z_{1}, \theta\right)\right\}$,

(ii)

$$
s\left(z_{1}, z_{2}\right)=1, \quad \text { otherwise. }
$$

LEMMA 2.1. Let $z_{1}, z_{2} \in G$. Then

$$
s\left(z_{1}, z_{2}\right)=\min \left\{\frac{\left|z_{1}-z_{2}\right|}{\min \left[d\left(z_{1}\right), d\left(z_{2}\right)\right]}, 1\right\}
$$

(ii)

$$
s\left(z_{1}, z_{2}\right)=0 \quad \text { if and only if } z_{1}=z_{2} \text {, }
$$

(iii)

$$
s\left(z_{1}, z_{2}\right)=s\left(z_{2}, z_{1}\right)
$$

(iv) for any $z_{0} \in G$

$$
s\left(z_{0}, z_{1}\right)+s\left(z_{0}, z_{2}\right) \geq \frac{1}{4} s\left(z_{1}, z_{2}\right)
$$

(v) If $s\left(z_{1}, z_{2}\right)<1$, then for any point $z_{0}$ on the straight line joining $z_{1}$ to $z_{2}$, we have

$$
s\left(z_{1}, z_{0}\right)<1 \text {, and } s\left(z_{2}, z_{0}\right)<1 \text {. }
$$


Remark. (A) $s$ does not satisfy the triangular inequality (and we call it a neo-metric). For example, let $G=\{z ; \operatorname{Re}(z)>0\}$ and $z_{1}=4$, $z_{2}=8, z_{0}=5$, then $s\left(z_{0}, z_{1}\right)+s\left(z_{0}, z_{2}\right)=1 / 4+3 / 5=17 / 20<1=s\left(z_{1}, z_{2}\right)$.

(B) The number $1 / 4$ in (2.6) may not be best possible factor in the inequality.

Proof. We may suppose $d\left(z_{1}\right) \leq d\left(z_{2}\right)$. To show (2.3), if $\left|z_{1}-z_{2}\right| \geq$ $d\left(z_{1}\right)$, then $z_{2} \notin G\left(z_{1}, \theta\right)$ for each $0<\theta<1$ and we have $s\left(z_{1}, z_{2}\right)=1$. On the other hand, if $\left|z_{1}-z_{2}\right|<d\left(z_{1}\right)$, let $\theta_{0}=\left(\left|z_{1}-z_{2}\right| / d\left(z_{1}\right)\right)(<1)$, then for each $0<\delta<\theta_{0}$ such that $\theta_{0}+\delta<1, z_{2} \in G\left(z_{1}, \theta_{0}+\delta\right)$ and certainly $z_{1} \in$ $G\left(z_{2}, \theta_{0}+\delta\right)$; while $z_{2} \notin G\left(z_{1}, \theta_{0}-\delta\right)$. i.e., $\theta_{0}=\inf \left\{\theta: 0<\theta<1, z_{1} \in G\left(z_{2}, \theta\right)\right.$, $\left.z_{2} \in G\left(z_{1}, \theta\right)\right\}$. Hence (2.3) is valid.

(2.4) and (2.5) are trivial. We now start to show (2.6). Let $z_{0} \in G$, and without loss of generality, let $s\left(z_{1}, z_{0}\right)<1$, and $s\left(z_{2}, z_{0}\right)<1$. Hence $\left|z_{1}-z_{0}\right|<\min \left\{d\left(z_{0}\right), d\left(z_{1}\right)\right\}$ and $\left|z_{2}-z_{0}\right|<\min \left\{d\left(z_{0}\right), d\left(z_{2}\right)\right\}$. By the definition of $d(z)$ we have

$$
\begin{aligned}
d\left(z_{0}\right) & \leq d\left(z_{1}\right)+\left|z_{0}-z_{1}\right| \leq 2 d\left(z_{1}\right) \text { and } d\left(z_{2}\right) \leq d\left(z_{0}\right)+\left|z_{0}-z_{2}\right| \\
& \leq 2 d\left(z_{0}\right) \leq 4 d\left(z_{1}\right) .
\end{aligned}
$$

Hence

$$
\begin{aligned}
s\left(z_{0}, z_{1}\right)+s\left(z_{0}, z_{2}\right) & =\frac{\left|z_{1}-z_{2}\right|}{\min \left\{d\left(z_{0}\right), d\left(z_{1}\right)\right\}}+\frac{\left|z_{2}-z_{0}\right|}{\min \left\{d\left(z_{2}\right), d\left(z_{0}\right)\right\}} \\
& \geq \frac{\left|z_{1}-z_{0}\right|}{2 d\left(z_{1}\right)}+\frac{\left|z_{2}-z_{0}\right|}{4 d\left(z_{1}\right)} \geq \frac{\left|z_{1}-z_{0}\right|+\left|z_{2}-z_{0}\right|}{4 d\left(z_{1}\right)} \\
& \geq \frac{\left|z_{1}-z_{2}\right|}{4 d\left(z_{1}\right)} \geq \frac{1}{4} s\left(z_{1}, z_{2}\right)
\end{aligned}
$$

For (2.7), note that if $s\left(z_{1}, z_{2}\right)<1$, then $\max \left\{\left|z_{2}-z_{0}\right|,\left|z_{1}-z_{0}\right|\right\} \leq$ $\left|z_{1}-z_{2}\right|<\min \left\{d\left(z_{1}\right), d\left(z_{2}\right)\right\}$. Hence $d\left(z_{0}\right) \geq d\left(z_{1}\right)-\left|z_{1}-z_{0}\right|>\left|z_{1}-z_{2}\right|-\left|z_{1}-z_{0}\right|$ $\geq\left|z_{2}-z_{0}\right|$. i.e., $s\left(z_{2}, z_{0}\right)=\left(\left|z_{2}-z_{0}\right| / \min \left[d\left(z_{2}\right), d\left(z_{0}\right)\right]\right)<1$. Similarly, we can show $s\left(z_{1}, z_{0}\right)<1$.

In a sense, this neo-metric gives us a "refined" measurement when compared to the Euclidean metric in $G$ for points which are near to the boundary. It works almost like the non-Euclidean metric (see also the following results) in a simply-connected region. In fact, let $G=D$, and let $\left\{z_{n}\right\}$ be an increasing sequence of positive numbers such that $\lim z_{n}$ 
$=1$. If $\left(1-z_{n+1}\right) / 1-z_{n} \leq 1 / 2$, then $\lim \sup \rho\left(z_{n}, z_{n+1}\right) \geq 1 / 3$ and $s\left(z_{n}, z_{n+1}\right)$ $=1$ for each $n=1,2, \ldots$. Conversely, if $\lim s\left(z_{n}, z_{n+1}\right)=0$, then $\lim \left(1-z_{n+1}\right) / 1-z_{n}=1$, and we have (for example, see [3, Lemma 4]) $\lim \rho\left(z_{n}, z_{n+1}\right)=0$. To be more precise, in describing the relation between the non-Euclidean metric $\rho$ and the neo-metric $s$ in a simply-connected region of hyperbolic type, we shall prove the following results. First, we show the main lemma in this section.

LEMMA 2.2. Let $G$ be a simply-connected region of hyperbolic type in the $\zeta$ plane. For each $a \in G$, let $f_{a}$ be a one-to-one conformal mapping from $G$ onto $D$ such that $f_{a}(a)=0$. Then for each $\theta, 0<\theta<1$,

$$
\left\{|z|<\frac{\theta}{16}\right\} \subseteq f_{a}(G(a, \theta)) \subseteq\{|z|<\min (4 \theta, 1)\} .
$$

Proof. We will show $\{|z|<\theta / 16\} \subseteq f_{a}(G(a, \theta))$ first. Let $z=g(w)=$ $f_{a}(a+\theta d(a) w)$, then $g(w)$ is one-to-one on $|w|<1$. By Koebe's distortion theorem, $f_{a}(G(a, \theta))=g(|w|<1)$ covers $\left\{|z|<\left|g^{\prime}(0)\right| / 4\right\}$. Note that $g^{\prime}(w)$ $=\theta d(a) f_{a}^{\prime}(\zeta)$, where $\zeta=a+\theta d(a) w$. It follows that $\left|g^{\prime}(0)\right|=\theta d(a)\left|f_{a}^{\prime}(a)\right|$. Let $h$ be the inverse function of $f_{a}$, then $h$ is one-to-one on $|z|<1$. By a theorem of Seidel and Walsh [16, Theorem 3, p. 134], we have $D_{1}(\zeta)$ $\leq\left(1-|z|^{2}\right)\left|h^{\prime}(z)\right| \leq 4 D_{1}(\zeta)$, where $D_{1}(\zeta)$ is the radius of the largest smooth circle (boundary not included) with center at $\zeta$ and entirely contained in the Riemann configuration over the $\zeta$-plane onto which $\zeta=h(z)$ maps the region $|z|<1$. i.e., in our case, $D_{1}(a)=d(a)$. Hence, we have

$$
d(a) \leq \frac{1}{\left|f_{a}^{\prime}(a)\right|}=\left|h^{\prime}(0)\right| \leq 4 d(a) .
$$

Or, $\left|g^{\prime}(0)\right|=\theta d(a)\left|f_{a}^{\prime}(a)\right| \geq \theta / 4$. Thus $\left|g^{\prime}(0)\right| / 4 \geq \theta / 16$. It follows now that $f_{a}(G(a, \theta))=g\{|w|<1\}$ covers $\{|z|<\theta / 16\}$. To prove the second half of (2.8), i.e., to show $f_{a}(G(a, \theta)) \subset\{|z|<\min (1,4 \theta)\}$, we let $\theta<1 / 4$ and let $w=(h(z)-a) / d(a)=\{h(4 \theta \psi)-a\} / d(a)$. (i.e., $z=4 \theta \omega$.) It is easy to see that $k(\omega)$ is one-to-one on $|\omega|<1$. By Koebe's distortion theorem, $k(|\omega|<1)$ covers $\left\{|w|<k^{\prime}(0) / 4\right\}$. On the other hand, by the theorem of Seidel and Walsh which we quoted earlier (see (2.9)), $\left|k^{\prime}(0)\right|=4 \theta / d(a)$ $\times\left|h^{\prime}(0)\right| \geq 4 \theta / d(a) \cdot d(a)=4 \theta$. i.e., $k^{\prime}(0) / 4 \geq \theta$. Hence $k(|\omega|<1)$ covers $\{|w|<\theta\}$, that is equivalent to $h(|z|<4 \theta)$ covers $G(a, \theta)$ and our theorem is complete. 
COROLLARY 2.2.1. Let $G$ be a simply-connected region of hyperbolic type and $z_{0} \in G$. Then

(A) for each $R>0$, there exists a $\theta(0<\theta<1)$, depending only on $R$ (independent of the choice of $z_{0}$ ) such that $G\left(z_{0}, \theta\right) \subset D_{\rho}\left(z_{0}, R\right)$,

(B) if $R<1 / 2 \log 17 / 15$, then there exist $\theta_{1}$ and $\theta_{2},\left(0<\theta_{1}<\theta_{2}<1\right)$, depending on $R$ only, such that $G\left(z_{0}, \theta_{1}\right) \subset D_{\rho}\left(z_{0}, R\right) \subset G\left(z_{0}, \theta_{2}\right)$.

Proof. (A) Let $0<4 \theta<\left(e^{2 R}-1\right) /\left(e^{2 R}+1\right)<1$, then by Lemma 2.2, $f_{z_{0}}\left(G\left(z_{0}, \theta\right)\right) \subset\{|z|<4 \theta\}$. It is easy to see that by (1.1) we have $\{|z|<4 \theta\}$ $\subset D_{\rho}(0, R)$, i.e., $G\left(z_{0}, \theta\right) \subset D_{\rho}\left(z_{0}, R\right)$.

(B) If $R<1 / 2 \log 17 / 15$, then $\left(e^{2 R}-1\right) /\left(e^{2 R}+1\right)<1 / 16$. Choose $\theta_{2}$ such that $0<16\left(e^{2 R}-1\right) /\left(e^{2 R}+1\right)<\theta_{2}<1$, then by lemma 2.2 , we have $D_{\rho}(0, R) \subseteq\left\{|z|<\theta_{2} / 16\right\} \subset f_{z_{0}}\left(G\left(z_{0}, \theta_{2}\right)\right)$. The other half of part (B) follows from part (A) by choosing $\theta_{1}$ such that $0<4 \theta_{1}<\left(e^{2 R}-1\right) /\left(e^{2 R}+1\right.$.)

LEMMA 2.3. Let $z_{0} \in G$, and for $0<\theta \leq 1$, let

$$
D_{s}\left(z_{1}, \theta\right)=\left\{z \in G: s\left(z_{0}, z\right)<\theta\right\},
$$

then $G\left(z_{0}, \theta / 2\right) \subset D_{s}\left(z_{0}, \theta\right) \subset G\left(z_{0}, \theta\right)$.

Proof. If $s\left(z_{0}, z_{1}\right)<\theta \leq 1$, then $\left|z_{1}-z_{0}\right|<\theta d\left(z_{0}\right)$. Hence $z_{1} \in G\left(z_{0}, \theta\right)$. On the other hand, since $d\left(z_{0}\right) \leq d\left(z_{1}\right)+\left|z_{1}-z_{0}\right| \leq 2 d\left(z_{1}\right)$, we have

$$
\frac{2\left|z_{1}-z_{0}\right|}{d\left(z_{0}\right)} \geq \frac{\left|z_{1}-z_{0}\right|}{\min \left\{d\left(z_{1}\right), d\left(z_{0}\right)\right\}}=s\left(z_{1}, z_{0}\right) . \quad \text { i.e., if } z_{1} \in G\left(z_{0}, \theta / 2\right),
$$

then $\left|z_{1}-z_{0}\right|<(\theta / 2) d\left(z_{0}\right)$. Hence $s\left(z_{1}, z_{0}\right)<\theta$ and our proof is complete.

Combining Corollary 2.2.1 and lemma 2.3, we have the following connection between the non-Euclidean metric $\rho$ and $s$ in a simply-connected region as follows.

COROLLARY 2.3.1. Let $G$ be a simply-connected region of hyperbolic type and $z_{0} \in G$. Then

(A) for each $R>0$, there exists a $\theta(0<\theta<1)$, depending only on $R$ such that $D_{s}\left(z_{0}, \theta\right) \subset D_{\rho}\left(z_{0}, R\right)$.

(B) if $R<(1 / 4) \log 17 / 15$, then there exist $\theta_{1}, \theta_{2}\left(0<\theta_{1}<\theta_{2}<1\right)$, depending only on $R$ such that $D_{s}\left(z_{0}, \theta_{1}\right) \subset D_{\rho}\left(z_{0}, R\right) \subset D_{s}\left(z_{0}, \theta_{2}\right)$.

Remark. Suppose $G$ is a simply-connected region of hyperbolic type 
and $f$ is a meromorphic function in $G$. It is well known that $f$ is not normal in $G$ if and only if there exists a " $p$-sequence" (see Introduction) of $f(z)$ in $G$. i.e., there exists a boundary sequence $\left\{z_{n}\right\}$ of $G$ such that for each $R>0$, and for each infinite subsequence $\left\{z_{m}\right\}$ of $\left\{z_{n}\right\}, f(z)$ has Picard behavior in $\bigcup_{m=1}^{\infty} D_{\rho}\left(z_{m}, R\right)$. Here $\rho$ represents the non-Euclidean metric in $G$. However, it does not give us an explicit picture if $G$ is not as "simple" as a disk or a half plane because we do not know what a non-Euclidean disk would look like in $G$. But corollaries 2.2.1 and 2.3.1 give us a better understanding on the locations and relative sizes of these non-Euclidean disks. i.e., if $R<(1 / 4) \log 17 / 15$, for practical application, we could replace $D_{\rho}\left(z_{n}, R\right)$ by $G\left(z_{n}, \theta\right)$ or $D_{s}\left(z_{n}, \theta\right)$ for some suitable $\theta$ which depends only on $R$. And $G_{s}\left(z_{n}, \theta\right)$ or $D_{s}\left(z_{n}, \theta\right)$ are easy to recognize.

Before going on to the next section, we shall show that in the case when $G=D$, some improvements could be made on the results of the above corollaries.

Lemma 2.4. Let $z_{0} \in D$ and $0<\theta<1$. Then
(A) $D_{\rho}\left(z_{0}, \log (1+\theta)\right) \subset G\left(z_{0}, \theta\right) \subset D_{\rho}\left(z_{0}, \log \frac{1+\theta}{1-\theta}\right)$,
(B) $D_{\rho}\left(z_{0}, \log \left(1+\frac{\theta}{2}\right)\right) \subset D_{s}\left(z_{0},\right) \subset D_{\rho}\left(z_{0}, \log \frac{1+\theta}{1-\theta}\right)$.

Proof. (A) Without loss of generality, we may suppose that $z_{0}$ lies on the positive $x$-axis. Thus let $z_{0}=\gamma$ and let $a<\gamma<b$ such that $b-\gamma=$ $\gamma-a=\theta(1-\gamma)$. Suppose $\rho_{1}=\min \{\rho(a, \gamma), \rho(b, \gamma)\}$ and $\rho_{2}=\max \{\rho(a, \gamma)$, $\rho(b, \gamma)\}$, then $D_{\rho}\left(z_{0}, \rho_{1}\right) \subset G\left(z_{0}, \theta\right) \subset D_{\rho}\left(z_{0}, \rho_{2}\right)$. By an easy calculation, we have $\rho(a, \gamma)=\log \{(1+\theta)(1+\gamma) /((1+\gamma)-\theta(1-\gamma))\}$ and $\rho(b, \gamma)=\log \{((1+\gamma)+$ $\theta(1-\gamma)) /(1+\gamma)(1-\theta)\}$. Thus $\log (1+\theta) \leq \rho(r, a) \leq \log \{(1+\theta) /(1-\theta)\}$ and $\log (1 /(1-\theta)) \leq \rho(r, b) \leq \log \{(1+) \theta /(1-\theta)$.$\} i.e., \log (1+\theta) \leq \rho_{1} \leq$ $\rho_{2} \leq \log \{(1+\theta) /(1-\theta)\}$. In other words,

$$
D_{\rho}\left(z_{0}, \log (1+\theta)\right) \subset G\left(z_{0}, \theta\right) \subset D_{\rho}\left(z_{0}, \log \frac{1+\theta}{1-\theta}\right) .
$$

(B) follows immediately from (A) and lemma 2.3.

Remark. In $D, D_{s}\left(z_{0}, \theta\right)=\left\{z \in D: s\left(z, z_{0}\right)<\theta\right\}$, in general, is not a 
Euclidean disk (while $D_{\rho}\left(z_{0}, R\right)$ is always a Euclidean disk, though it may not have $z_{0}$ as its center). For example, let $z_{0}=1 / 2, \theta=1 / 2$, then

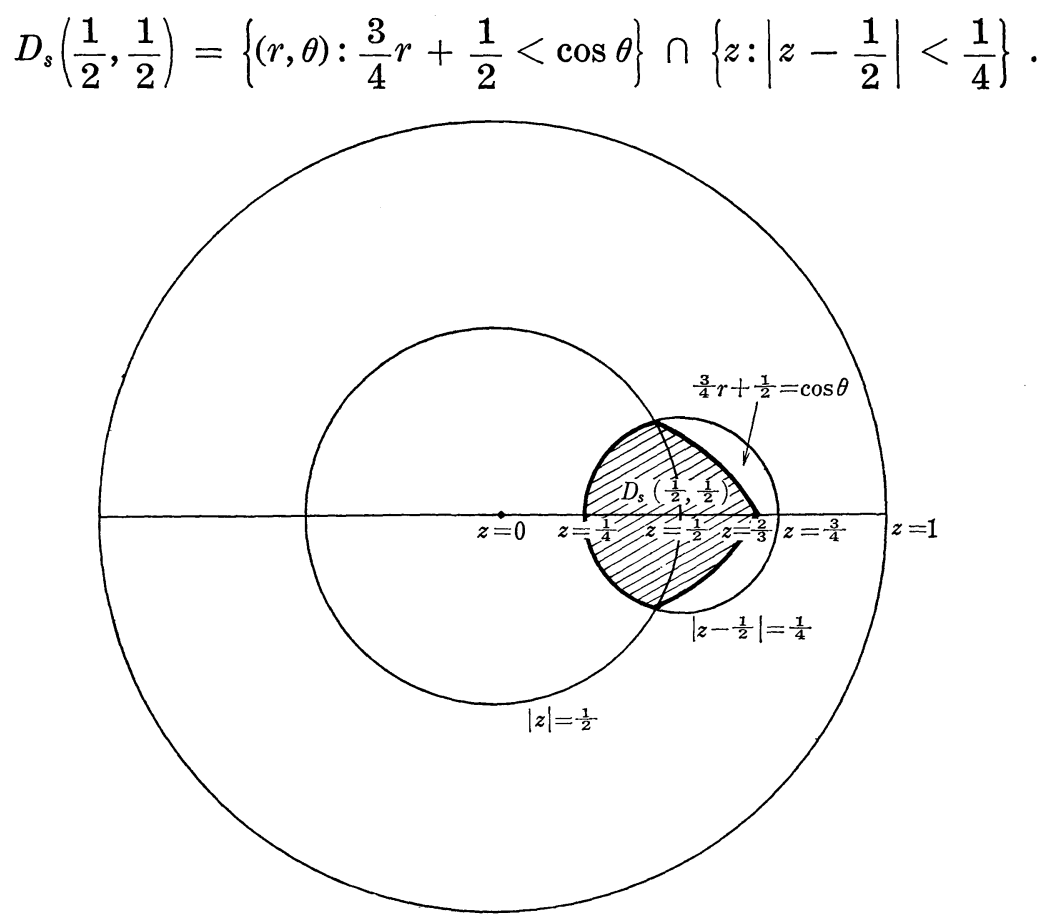

\section{Neo-Normal Functions in $G$}

DEFINITION 3.1. We say that a meromorphic function $f(z)$ is neonormal in $G$ if the family of functions $F=\left\{g_{z}(w)=f(z+d(z) w): z \in G\right\}$ is normal in the sense of Montel in $|w|<1$. (We shall use $s$-normal instead of neo-normal in the following discussions.)

THEOREM 3.1. $f(z)$ is s-normal in $G$ if and only if

$$
\sup \{d(z) \rho(f(z)): z \in G\} \leq K<\infty .
$$

Proof. Note that $(d / d w) g_{z}(w)=f^{\prime}(\omega) d(z)$, where $\omega=z+d(z) w$. If $f(z)$ is $s$-normal in $G$, i.e., $F$ is normal in $|w|<1$, by Marty's criterion on normalcy, $d(z) \rho(f(z))=\rho\left(g_{z}(0)\right) \leq K<\infty$ for each $z \in G$. Hence (3.1) is valid.

Conversely, if (3.1) is valid, we will show $F$ is normal in $|w|<1$. It suffices to show $F$ is normal in $|w| \leq \theta$ for each $0<\theta<1$. Fixed $z_{0} \in G$, if $\left|w_{1}\right| \leq \theta$ and if $z_{1}=z_{0}+d\left(z_{0}\right) w_{1}$, then $\left|z_{1}-z_{0}\right|=d\left(z_{0}\right)\left|w_{1}\right| \leq$ 
$\theta d\left(z_{0}\right)$. Since $d\left(z_{0}\right) \leq d\left(z_{1}\right)+\left|z_{1}-z_{0}\right| \leq d\left(z_{1}\right)+\theta d\left(z_{0}\right)$, thus $(1-\theta) d\left(z_{0}\right) \leq$ $d\left(z_{1}\right)$. On the other hand, $\rho\left(g_{z_{0}}\left(w_{1}\right)\right)=d\left(z_{0}\right) \rho\left(f\left(z_{1}\right)\right) \leq\left(d\left(z_{1}\right) /(1-\theta)\right) \rho\left(f\left(z_{1}\right)\right)<$ $K /(1-\theta)$, where $K$ is the bound in (3.1), i.e., $\sup _{|w| \leq \theta<1, z \in G}\left\{\sup _{\mid} \rho\left(g_{z}(w)\right\} \leq K /(1-\theta)\right.$. Hence $F$ is normal in $|w| \leq \theta$ for each $0<|w|<1$ and our proof is complete.

With the help of the neo-metric $s$, we will generalize the notion of " $p$-sequence" as follows.

DeFinition 3.2. A boundary sequence $\left\{z_{n}\right\}$ in $G$ is said to be an "s-sequence" of a meromorphic function $f(z)$ in $G$ if for each $0<\theta<1$, and for each infinite subsequence $\left\{z_{m}\right\}$ of $\left\{z_{n}\right\}, f(z)$ has Picard behavior in $\bigcup_{n=1}^{\infty} D_{s}\left(z_{m}, \theta\right)$.

THEOREM 3.2. A meromorphic function $f(z)$ in $G$ is s-normal if and only if it does not possess any s-sequence. (See also [4], [5] and [9].)

Proof. Suppose that $f(z)$ is not s-normal in G. By theorem 3.1, there exists a boundary sequence $\left\{z_{n}\right\}$ in $G$ such that $\lim d\left(z_{n}\right) \rho\left(f\left(z_{n}\right)\right)=\infty$. Write $g_{n}(w)=f\left(z_{n}+d\left(z_{n}\right) w\right)$, then $\lim \rho\left(g_{n}(0)\right)=\lim d\left(z_{n}\right) \rho\left(f\left(z_{n}\right)\right)=\infty$. i.e., $\left\{g_{n}(w)\right\}$ is not normal in $|w|<\theta$ for each $0<\theta<1$. In particular, $\left\{g_{n}(w)\right\}$ cannot omit three values there. Hence $f(z)$ has Picard behavior in $\bigcup_{n=1}^{\infty} D_{s}\left(z_{n}, \theta\right)$ for each $0<\theta<1$, which is equivalent to saying that $\left\{z_{n}\right\}$ is an $s$-sequence.

Conversely, suppose that $f(z)$ is $s$-normal and that it possesses an $s$-sequence $\left\{z_{n}\right\}$. We may let $\lim f\left(z_{n}\right)=a$ exist. By definition 3.1 , the sequence of functions $\mathscr{F}=\left\{g_{n}(w)=f\left(z_{n}+d\left(z_{n}\right) w\right): n=1,2, \cdots\right\}$ is normal in $|w|<1$. Hence it is $(d-\chi)$ equicontinuous in $|w| \leq \theta$, for each $0<\theta$ $<1$. (For example, see [10, Sect. 2, p, 155].) Choose $\theta$, such that $\chi\left(g_{m}(w), g_{m}(0)\right)<1 / 4$ for all $|w| \leq 2 \theta<1$, i.e., $\chi\left(f\left(z_{m}\right), f(z)\right)<1 / 4$ for all $z \in D\left(z_{m}, \theta\right)$. Hence, for sufficiently large $n, \sup \left\{\chi(f(z), a): z \in D_{s}\left(z_{n}, \theta\right)\right\}$ $<1 / 2$, i.e., $f(z)$ does not have Picard behavior in $\bigcup_{n=1}^{\infty} D_{s}\left(z_{n}, \theta\right)$, which contradicts our assumption that $\left\{z_{n}\right\}$ is an $s$-sequence and our proof is now complete.

Remark. It is clear that if $G$ is a simply-connected region of hyperbolic type, from the viewpoint of theorems $3.1,3.2$; or corollary 2.3.1, that the definitions of "normal function" in [12] and [14] are equivalent to that of "s-normal function." Meanwhile, the definitions of " $p$-sequence" 
in [4], [5] and [9] are equivalent to that of $s$-sequence. However, as we will see later in section 5 , if $G$ is multiply-connected, the definition of "normal functions" in [12] is not the same as that of " $s$-normal functions" here.

We shall list some results concerning s-normal functions defined in an arbitrary region. These results have been proved for the case when $G=D$. (For example, see [1].) In most instances, the proofs are essentially the same as those for the disk and we will omit them.

LEMMA 3.3. Let $f(z)$ be meromorphic and s-normal in $G$ and let $\left\{z_{n}\right\},\left\{z_{n}^{\prime}\right\}$ be two boundary sequences in $G$.

(A) If $\left\{f\left(z_{n}\right)\right\}$ is bounded away from $w \in \Omega$, then there exists a $\theta$ $(0<\theta<1)$, such that $f(z)$ is bounded away from $w$ in $\bigcup_{n=1}^{\infty} D_{s}\left(z_{n}, \theta\right)$.

(B) If $\lim f(z)=w, w$ being assumed by $f(z)$ only a finite number of times, then $f(z)$ tends to $w$ uniformly in $\bigcup_{n=1}^{\infty} D_{s}\left(z_{n}, \theta\right)$ for each $0<\theta<1$.

(C) If $\lim s\left(z_{n}, z_{n}^{\prime}\right)=0$, then $\lim f\left(z_{n}\right)=a$ if and only if $\lim f\left(z_{n}^{\prime}\right)$ $=a$.

\section{Application-the Growth of Derivatives}

Generalizing the definition of regular sequence of Seidel and Walsh [16, pp. 153-54], we have

DEFINITION 4.1. Let $f(z)$ be a meromorphic function and let $\left\{z_{n}\right\}$ be a boundary sequence in $G$. If there exists a $\theta_{0}, 0<\theta_{0}<1$, such that for any sequence of points $\left\{z_{n}^{\prime}\right\}$ with $\lim \sup s\left(z_{n}, z_{n}^{\prime}\right)<\theta_{0}$, we have

$$
\lim \chi\left[f\left(z_{n}\right), f\left(z_{n}^{\prime}\right)\right]=0,
$$

then we call $\left\{z_{n}\right\}$ a chordally s-regular sequence of $f(z)$.

If instead of (4.1) we have

$$
\lim \left|f\left(z_{n}\right)-f\left(z_{n}^{\prime}\right)\right|=0,
$$

then we call $\left\{z_{n}\right\}$ an $s$-regular sequence of $f(z)$.

DEFINITION 4.2. ([16, p. 154]) Let $f(z)$ be meromorphic and let $\left\{z_{n}\right\}$ be a boundary sequence in $G$. If for each integer $1 \leq k \leq m$, we have

$$
\lim _{n \rightarrow \infty} d\left(z_{n}\right)^{k} f^{(k)}\left(z_{n}\right)=0
$$


while

$$
\lim \sup d\left(z_{n}\right)^{m+1}\left|f^{(m+1)}\left(z_{n}\right)\right|>0,
$$

then we call $\left\{z_{n}\right\}$ a quasi-regular sequence of order $m$.

The case $m=\infty$ is allowed and means $\lim _{n \rightarrow \infty} d\left(z_{n}\right)^{k} f^{(k)}\left(z_{n}\right)=0$ for each positive integer $k$.

Remark. The notion of chordally regular sequence is also used in the study of value distribution of certain class of meromorphic functions, for example, for those which do not possess any chordally regular sequence in the unit disk. For some detail on this related problem, see [17].

We are going to investigate the necessary and sufficient conditions for a boundary sequence to be quasi-regular of infinite order. There are two approaches, one employed by Eggleton [2] and the other by Seidel and Walsh [16]. We shall generalize Eggleton's method first.

LEMma 4.1. Let $f(z)$ be a meromorphic function and let $\left\{z_{n}\right\}$ be a boundary sequence in $G$. Suppose that

(1) $\lim _{n \rightarrow \infty} f\left(z_{n}\right)=0$

(2) for some $0<\theta<1, f(z)$ is bounded in

$$
G_{1}(\theta)=\bigcup_{n=1}^{\infty} G\left(z_{n}, \theta\right)
$$

and

(3) for each $0<\theta<\theta_{1}$, and for each $n=1,2, \ldots$

there exists $z_{n}^{\prime},\left|z_{n}^{\prime}-z_{n}\right|=\theta d\left(z_{n}\right)$ such that

$$
\lim _{n \rightarrow \infty} f\left(z_{n}^{\prime}\right)=0 \text {. }
$$

Then $\left\{z_{n}\right\}$ is a quasi-regular sequence of infinite order. In fact, we have

$$
\lim _{n \rightarrow \infty} d\left(z_{n}\right)^{k} f^{(k)}\left(z_{n}\right)=0 \quad \text { for } k=0,1,2, \cdots
$$

Proof. Let the bound of $f(z)$ in $G(\theta)$ be $M$. Write $\Gamma_{n}=\partial G\left(z_{n}, \theta_{1} / 2\right)$, then

$$
f^{(k)}\left(z_{n}\right)=\frac{k !}{2 \pi i} \int_{\Gamma_{n}} \frac{f(\zeta)}{\left(\zeta-z_{n}\right)^{k+1}} d \zeta
$$




\section{Hence}

(4.8) $\left|f^{k}\left(z_{n}\right)\right| \leq \frac{k ! M}{\left(\left(\theta_{1} / 2\right) d\left(z_{n}\right)\right)^{k}}, \quad$ for $k=0,1, \cdots$, and $n=1,2, \cdots$

We shall show by induction that

$$
\lim _{n \rightarrow \infty} d\left(z_{n}\right)^{k}\left|f^{(k)}\left(z_{n}\right)\right|=0 \quad \text { for } k=0,1,2, \cdots,
$$

When $k=0$, (4.5) reduces to $\lim _{n \rightarrow \infty} f\left(z_{n}\right)=0$, which is a part of our hypothesis. Suppose now

$$
\lim _{n \rightarrow \infty} f^{(k)}\left(z_{n}\right) d\left(z_{n}\right)^{k}=0 \quad \text { for } k=0,1,2, \cdots, K-1,
$$

By Taylor's formula

$$
f\left(z_{n}^{\prime}\right)=\sum_{k=0}^{\infty} \frac{\left(z_{n}^{\prime}-z_{n}\right)^{k}}{k !} f^{(k)}\left(z_{n}\right)
$$

\section{Hence}

$$
\frac{\left(z_{n}^{\prime}-z_{n}\right)^{K} f^{(K)}\left(z_{n}\right)}{K !}=f\left(z_{n}^{\prime}\right)-\sum_{k=0}^{K-1} \frac{\left(z_{n}^{\prime}-z_{n}\right)^{k}}{k !} f^{(k)}\left(z_{n}\right)-\sum_{k=K+1}^{\infty} \frac{\left(z_{n}^{\prime}-z_{n}\right)^{k}}{k !} f^{(k)}\left(z_{n}\right) .
$$

And

$$
\begin{aligned}
\frac{\left|z_{n}^{\prime}-z_{n}\right|^{K}\left|f^{(K)}\left(z_{n}\right)\right|}{K !} \leq\left|f\left(z_{n}^{\prime}\right)\right| & +\sum_{k=0}^{K-1} \frac{\left|z_{n}^{\prime}-z_{n}\right|^{k}}{k !}\left|f^{(k)}\left(z_{n}\right)\right| \\
& +\sum_{k=K+1}^{\infty} \frac{\left|z_{n}^{\prime}-z_{n}\right|^{k}}{k !}\left|f^{(k)}\left(z_{n}\right)\right|
\end{aligned}
$$

i.e.,

$$
\begin{aligned}
\frac{\left(\theta d\left(z_{n}\right)\right)^{K}\left|f^{(K)}\left(z_{n}\right)\right|}{K !} \leq\left|f\left(z_{n}^{\prime}\right)\right| & +\sum_{k=0}^{K-1} \frac{\theta^{k}}{k !} d\left(z_{n}\right)^{k}\left|f^{(k)}\left(z_{n}\right)\right| \\
& +\sum_{k=K+1}^{\infty} \frac{\theta^{k} d\left(z_{n}\right)^{k}}{k !}\left|f^{(k)}\left(z_{n}\right)\right| .
\end{aligned}
$$

Thus, by (4.8),

$$
\begin{aligned}
\frac{\theta^{K}}{K !} \lim _{n \rightarrow \infty} d\left(z_{n}\right)^{K}\left|f^{(K)}\left(z_{n}\right)\right| \leq \lim _{n \rightarrow \infty}\left|f\left(z_{n}^{\prime}\right)\right| & +\sum_{k=0}^{K-1} \frac{\theta^{k}}{k !} \lim _{n \rightarrow \infty} d\left(z_{n}\right)^{k}\left|f^{(k)}\left(z_{n}\right)\right| \\
& +\sum_{k=K+1}^{\infty}\left(\frac{2 \theta}{\theta_{1}}\right)^{k} M .
\end{aligned}
$$

And by (4.5), (4.6) and (4.9), we have 


$$
\begin{aligned}
\lim _{n \rightarrow \infty} d\left(z_{n}\right)^{K}\left|f^{(K)}\left(z_{n}\right)\right| \leq \frac{M K !}{\theta^{K}} \sum_{k=K+1}^{\infty}\left(\frac{2 \theta}{\theta_{1}}\right)^{k} & =\frac{M K !}{\theta^{K}} \frac{\left(2 \theta / \theta^{1}\right)^{K+1}}{1-\left(2 \theta / \theta_{1}\right)} \\
& =2\left(\frac{2}{\theta_{1}}\right)^{K} \frac{M K !}{\theta_{1}-2 \theta} \cdot \theta
\end{aligned}
$$

Letting $\theta \rightarrow 0$ we have $\lim _{n \rightarrow \infty} d\left(z_{n}\right)^{K}\left|f^{(K)}\left(z_{n}\right)\right|=0$ and our induction process is complete.

CoRollary 4.1.1. If $f(z)$ is s-normal and meromorphic in $G, L$ is a boundary path in $G$ such that

$$
\lim _{\substack{z \rightarrow \partial G \\ z \in L}} f(z)=b \neq \infty
$$

then $\lim _{\substack{z \rightarrow \partial G \\ z \in L}} f^{(k)}(z) d(z)^{k}=0$ for $k=1,2, \cdots$

Proof. Without loss of generality, let $b=0$, for otherwise, we may consider $f(z)-b$. By Lemma 3.3, $f(z)$ is bounded in $\bigcup_{z \in L} D_{s}\left(z_{1}, \theta\right)$ for some $0<\theta<1$. Our corollary follows immediately from Lemma 4.1.

Remark. If $b=\infty$, the above corollary is false. (See also the remark following Lemma 4.2.)

COROLlaRY 4.1.2. Let $f(z)$ be s-normal, holomorphic and have only a finite number of zeros in $G$. If $S$ is any set in $G$ such that $\bar{S} \cap \partial G$ $\neq \emptyset$ and $\lim _{\substack{z \rightarrow \partial G \\ z \in S}} f(z)=0$, then

$\lim _{z \rightarrow \bar{S} \cap \partial G} d(z)^{k} f^{(k)}(z)=0$ for each $k=1,2, \cdots$, and for each $0<\theta<1$, where $S(\theta)=\bigcup_{z \in S} D_{s}(z, \theta)$.

Proof. From Lemma 3.3, $f(z)$ tends to zero uniformly in $S(\theta)$ and our result is an immediate consequence of Lemma 4.1.

COROLlaRY 4.1.3. If $f(z)$ is meromorphic and assumes every value in $\Omega$ at most a finite number of times in $G$, then each boundary sequence $\left\{z_{n}\right\}$ in $G$ such that $\lim \sup \left|f\left(z_{n}\right)\right| \neq \infty$, is a quasi-regular sequence of infinite order.

Proof. Trivial!

COROLLARY 4.1.4. Let $f(z)$ be s-normal and meromorphic and let $\left\{z_{n}\right\}$ 
be a boundary sequence in $G$ such that $\lim s\left(z_{n}, z_{n+1}\right)=0$ and $\lim f\left(z_{n}\right)$ $=b \neq \infty$. Then $\left\{z_{n}\right\}$ is a quasi-regular sequence of infinite order.

Proof. Let $L$ be the boundary path in $G$ formed by joining consecutive points $z_{n}$ to $z_{n+1}$ by an Euclidean straight line. By Lemma 2.1 and Lemma 3.3, $\lim _{\substack{z \rightarrow a G \\ z \in L}} f(z)=b \neq \infty$. Our corollary now follows directly from Corollary 4.1.3.

COROLlaRY 4.1.5. Let $f(z)$ be meromorphic and s-normal and omit two values in $G$. If $\left\{z_{n}\right\}$ is a boundary sequence in $G$ such that $\lim \sup s\left(z_{n}, z_{n+1}\right)<1$ and $\lim _{n \rightarrow \infty} f\left(z_{n}\right)=w$, where $w$ is one of the omitted values, then $\left\{z_{n}\right\}$ is a quasi-regular sequence of infinite order.

Proof. Same as Corollary 4.5.

Remark. Corollary 4.1.1 generalizes [2, Theorem 1]; Corollary 4.1.2 generalized Theorems 1 and 3 and Corollary 1 in [18]; Corollary 4.1.3 generalizes Corollaries 7 and 8 in [18] and Corollary 4.1.5 generalizes Corollaries 2, 3, 4, 5 and 6 in [18].

Now, we are going to look into the same problem from the viewpoint employed by Seidel and Walsh [16].

LEMMA 4.2. A necessary and sufficient condition that $\left\{z_{n}\right\}$ be an $s$ regular sequence for a holomorphic s-normal function $f(z)$ in $G$ is that it be quasi-regular of infinite order.

Proof. The condition is necessary. If $\left\{z_{n}\right\}$ is an $s$-regular sequence of $f(z)$, then there exists a positive number $\theta$, such that $2 \theta(0<2 \theta<1)$ is the $\theta_{0}$ in Definition 4.1.

Consider the sequence of functions

$$
\left\{g_{n}(\zeta)=f\left(z_{n}+\theta d\left(z_{n}\right) \zeta\right)-f\left(z_{n}\right)\right\} .
$$

Since $f(z)$ is $s$-normal, thus $\left\{g_{n}(\zeta)\right\}$ is normal in $|\zeta|<1$. We shall show $\lim g_{n}(\zeta)=0$ uniformly in some disk $|\zeta| \leq \lambda<1$. If $|\zeta| \leq \lambda$ and $z=z_{n}+\theta d\left(z_{n}\right) \zeta$, then $\left|z-z_{n}\right| \leq \lambda \theta d\left(z_{n}\right)$. i.e., $s\left(z_{n}, z\right) \leq 2 \lambda \theta<2 \theta$. Given any $\varepsilon<0$, by the definition of $s$-regular sequence, there exists an integer $N(\varepsilon)$ such that for all $n<N(\varepsilon)$ and $z \in D_{s}\left(z_{n}, 2 \theta\right)$, we have $\left|f(z)-f\left(z_{n}\right)\right|$ $<\varepsilon$. i.e., for $|\zeta| \leq \lambda,\left|g_{n}(\zeta)\right|<\varepsilon$. Hence $\lim _{n \rightarrow \infty} g_{n}(\zeta)=0$ uniformly in $|\zeta| \leq \lambda$. This implies that $\lim _{n \rightarrow \infty} g_{n}^{(k)}(\zeta)=0$ for each $k=1,2, \cdots$, and for 
$|\zeta|<\lambda$. Since $g_{n}^{(k)}(0)=\theta^{k} d\left(z_{n}\right)^{k} f^{(k)}\left(z_{n}\right)$, i.e., $\lim _{n \rightarrow \infty} d\left(z_{n}\right)^{k} f^{(k)}\left(z_{n}\right)=0$, for $k=$ $1,2, \cdots$ Thus $\left\{z_{n}\right\}$ is quasi-regular of infinite order.

Conversely, since $\left\{g_{n}(\zeta)\right\}$ is normal in $|\zeta|<1$, then a suitable subsequence of $\left\{g_{n}(\zeta)\right\}$ would converge uniformly in each disk $|\zeta| \leq \lambda<1$ to some holomorphic function $G(\zeta)$ for which $G(0)=0$. Hence $G(\zeta)=$ $c_{1} \zeta+c_{2} \zeta^{2}+\cdots \quad$ On the other hand, $g_{n}(\zeta)=\sum_{k=1}^{\infty}\left(\left(\theta d\left(z_{n}\right)\right)^{k} / k !\right) f^{(k)}\left(z_{n}\right) \zeta^{k}$. Hence $\lim _{n \rightarrow \infty}\left(\theta^{k} / k !\right) d\left(z_{n}\right)^{k} f^{(k)}\left(z_{n}\right)=c_{n}$. It is clear now if $\left\{z_{n}\right\}$ is quasi-regular of infinite order, then $G(\zeta) \equiv 0$ in each disk $|\zeta| \leq \lambda<1$, and hence $\left\{z_{n}\right\}$ is $s$-regular.

Remark. (A) As shown above, because of the strong hypothesis for regular sequence, we do not need the boundedness of $\left\{f\left(z_{n}\right)\right\}$ to prove Lemma 4.2, Seidel and Walsh had overlooked this point in the proofs of [16, Theorem 5, p. 156] and [16, Corollary 4, p. 198]. However, if $\left\{z_{n}\right\}$ is only chordally $s$-regular, the necessity in Lemma 4.2 is not valid. For example, let $f(z)=1 /(1-z)$, then it is holomorphic and normal in $D$, but $\lim (1-r) f^{\prime}(r)=\infty$. In such case, the boundedness of $\left\{f\left(z_{n}\right)\right\}$ is needed.

(B) All the corollaries of Lemma 4.1 are also corollaries of Lemma 4.2. It is because the hypothesis of each corollary would imply that the boundary sequence (in consideration) is $s$-regular and that $f(z)$ is holomorphic in some neighborhood of the boundary sequence.

(C) By Definition 3.2, and by Theorems 3.1, 3.2, and the fact that $d(z)\left|f^{\prime}(z)\right| \geq d(z) \rho(f(z))$, it is easy to see that a necessary condition for a boundary sequence $\left\{z_{n}\right\}$ in $G$ to be an $s$-regular (chordally $s$-regular) or quasi-regular of order $k, k \geq 1$, of $f(z)$ is that $\left\{z_{n}\right\}$ is not an $s$-sequence of $f(z)$. On the other hand, Lemma 4.1, its corollaries, and Lemma 4.2 remain valid if we replace the hypothesis that $f(z)$ is $s$-normal in $G$ by that the boundary sequence(s) in consideration is not an $s$-sequence of $f(z)$.

\section{Some Remarks}

We wish to remark here about the connections between our results and those of Lehto [11], Lehto and Virtanen [12], and Gavrilov [6], [7] and [8] concerning meromorphic function defined in a deleted neighborhood of one of its essential singularities. First of all, we examine the following definition and lemma in [11]. 
DEFINITION 5.1. A meromorphic function $f(z)$ defined in a domain $G$ is said to be weakly normal if $f(z)$ is normal in every simply-connected subregion of $G$.

LEMMA 5.1. Let $f(z)$ be meromorphic in a deleted neighborhood $G$ of its essential singularity $z=0$. Then the following are true.

(A) $f(z)$ is weakly normal in $G$ if and only if

$$
\limsup _{z \rightarrow 0}|z| \rho(f(z))<\infty,
$$

(B) if $f(z)$ omits one value in $U$, then

$$
\limsup _{z \rightarrow 0}|z| \rho(f(z))=\infty .
$$

Applying our notion, we have

COROLlaRY 5.1.1. Let $f(z)$ be a meromorphic function defined in a region $G$.

(A) If $G$ is simply-connected, then $f(z)$ is normal in $G$ if and only if it is weakly normal in $G$, if and only if it is s-normal in $G$.

(B) If $G$ is a deleted neighborhood of $z=0$, an essential singularity of $f(z)$, then $f(z)$ is weakly normal in $G$ if and only if $f(z)$ is s-normal in $G$, if and only if

$$
\limsup _{z \rightarrow 0}|z| \rho(f(z))<\infty .
$$

(C) If $f(z)$ and $G$ are defined as in (B), and if in addition, $f(z)$ omits one value in $G$, then $f(z)$ is not s-normal in $G$.

Proof. Part (A) follows directly from Theorem 3.2 and the fact that the notions "normal" and "weakly normal" are equivalent in a simply-connected region.

Part (B) follows part (A) of Lemma 5.1 and the fact that $d(z)=|z|$.

Part (C) follows from part (B) of Lemma 5.1 and part (B) of this corollary.

Remark. From the above corollary, we see that a meromorphic function could be $s$-normal in a deleted neighborhood of an essential singularity while Lehto and Virtanen [12] showed $f(z)$ could never be normal there. In this case, "normal" always implies "weakly normal" which is equivalent to " $s$-normall" by part (B) of the above corollary. 
The author is not so sure what we could say about the implication going in the other direction.

Another point of interest is the theorem investigated by Lehto [11] and later by Gavrilov [7] and [8]. In fact, using our notions, we have the following theorem for which the proof will be omitted.

THEOREM 5.2. Let $h(r)>0$ be an arbitrary real-valued function defined for all $r>0$ such that $h(r) \leq r$, and let $f(z)$ be a meromorphic function in $G$. If $\left\{z_{n}\right\}$ is a boundary sequence such that

$$
\lim _{n \rightarrow \infty} h\left(d\left(z_{n}\right)\right) \rho\left(f\left(z_{n}\right)\right)=\infty,
$$

then $f(z)$ has Picard behavior in $\bigcup_{n=1}^{\infty} G\left(z_{n}, \delta p\left(d\left(z_{n}\right)\right)\right.$ for each $\delta>0$, where $p(r)=h(r) / r . \quad$ In particular, when $h(r)=r$, we have Theorem 3.2.

\section{Acknowledgment}

We wish to thank Professor S. W. Chan for helpful suggestions, and Dr. P. Gauthier for the corrections of the mistakes in the preprint of this paper.

\section{REFERENCES}

[1] Bagemihl, F., and Seidel, W., Sequential and continuous limits of meromorphic functions, Ann. Acad. Sci. Fenn. AI 280 (1960), pp. 1-17.

[2] Eggleston, H., A Tauberian theorem, Proc. London Math. Soc. (3) 1 (1951), pp. 28-45.

[3] Gauthier, P., Cercles de remplissage and spiral asymptotic behavior, to appear in Pacific J. M.

[4] - A criterion on normalcy, Nagoya Math. J. 32 (1968), pp. 277-282.

[ 5 ] Gavrilov, V. I., On the distribution of values of non-normal meromorphic functions in the unit disc, (in Russian) Mat. Sb. 109 (n.s. 67) (1965), pp. 408-427.

[6] — The behavior of a holomorphic function near an essential singularity, (in Russian) Dokl. Akad. Nauk SSSR 162 (1965), pp. 491-495. English translation: Soviet Math. Dokl. 6 (1965), pp. 693-696.

[7] - The behavior of meromorphic functions in the neighborhood of an essential singularity, Research report No. 2, Indian Institute of Technology, Bombay, India (1969), pp. 1-8.

[8] - The distribution of the values of meromorphic functions in the unit disk, Reseach report No. 3, Inidan Institute of Technology, Bombay, India (1969), pp. 1-8. Also appeared in AMS Notices 16, No. 2-3 (1969).

[9] Lange, H., Sur les cercles de remplissage non-euclideans. Ann. Sci. École Norm. Sup. (3) 77 (1960), pp. 257-260.

[10] Lappan, P., Some results on harmonic functions, Math. Z. 90 (1965) pp. 155159. 
[11] Lehto, O., The spherical derivative of meromorphic function in the neighborhood of an isolated singularity, Comment. Math. Helv. 33 (1959), pp. 196-205.

[12] Lehto, O., and Virtanen, V. I., Boundary behavior and normal meromorphic functins, Acta Math. 97 (1957), pp. 47-65.

[13] — On the behavior of meromorphic functions in the neighborhood of an isolated singularity, Ann. Acad. Sci. Fenn. AI 240 (1959), pp. 1-9.

[14] Noshiro, K., Contributions to the theory of meromorphic functions in the unit circle, F. Fac. Sci. Hokkaido Univ. 7 (1938), pp. 149-159.

[15] Rogosinski, W., On the order of the derivatives of a function analytic in an angle, J. London Math. Soc. 20 (1945), pp. 100-109.

[16] Seidel, W., and Walsh, J. L., On the derivatives of functions analytic in the circle and their radii of univalence and p-valence, Trans. Amer. Math. Soc. 52 (1942), pp. 128-216.

[17] Tse, K. F., Some results on value distribution of meromorphic function in the unit disk, Nagoya Math. J. 34 (1969), pp. 105-119.

[18] Xou, X. T. (Tsou, H. T.), Concerning the properties of bounded regular functions near the boundary, Acta Math. Scinica (8) 3 (1958), pp. 396-405. English translation: Chinese Math. Acta (9) 1 (1967), pp. 106-115.

[19] Yosida, K., On a class of meromorphic functions, Proc. Phys.-Math. Sci. Japan 3 ser. 16 (1934), pp. 227-235.

Syracuse University 